\title{
Le TARMED à Genève
}

Nous publions ici un résumé d'une étude réalisée dans le canton de Genève entre mai et novembre 2001 intitulée «Etude d'impact de l'introduction de TARMED dans le domaine de la chirurgie ambulatoire et de la médecine d'investigation dans le canton de Genève» [1].

Nous publions également une lettre ouverte du Dr R. Nyffeler, président de la société médicale du Canton de Fribourg, adressée au comité de pilotage de cette étude ainsi que la réplique de celui-ci.

\section{Résumé}

mt. Cette étude, qui selon ses propres termes était «souhaitée par l'Etat de Genève et par tous les partenaires de la chirurgie ambulatoire, c'est-à-dire les assureurs maladies, les hôpitaux, l'association des Médecins du Canton de Genève», devait permettre aux prestataires de chirurgie ambulatoire du canton de défendre si nécessaire une valeur de point suffisante pour maintenir, voire encourager le développement de la chirurgie ambulatoire dans le canton de Genève. Le département de l'Action Sociale et de la Santé, représenté par la Direction générale de la santé, a réuni un comité de pilotage «pour assurer un consensus et une acceptation aussi large que possible des résultats de l'étude».

Ce comité était composé des personnes suivantes: Dr Annie Mino, directrice de la Santé; Mme Pauline de Vos Bolay, cheffe du service Tarifs et Clientèle des Hôpitaux Universitaires de Genève; Nicolas F. Froelicher, président de l'Association des Cliniques privées de Genève (ACPG); Mme Thérèse Laverrière, directrice du Service de l'assurance-maladie; Bernard Markwalder, président de la Fédération genevoise des assureurs-maladie; Dr Philippe Rheiner, chirurgien, représentant de l'Association des médecins du canton de Genève (AMG); Dr Jacques Alain Witzig, chirurgien, représentant de l'Association des médecins du canton de Genève (AMG).

La méthodologie a consistée à définir un échantillon des interventions les plus fréquentes (52) dans les sept établissements privés participant à l'étude et l'hôpital cantonal, et de pratiquer une saisie prospective afin d'obtenir un échantillon représentatif par intervention et par établissement. La valeur du point nécessaire pour garantir une neutralité de facturation a été calculée. Comme base de calculation, les versions TARMED 1.0 et 1.1 ont été utilisées.

Au total, sur 983 cas remis par les cliniques, 52 ont dû finalement être retirés par manque de données fournies par l'opérateur, laissant 931 cas disponibles pour l'étude. La valeur du point pour respecter la neutralité des coûts du secteur de la chirurgie ambulatoire a été calculée à une valeur moyenne de Fr. 1.65 (fourchette entre 1.61 et 1.69). Les pertes de rémunération du secteur pour des valeurs de point dans la fourchette de l'étude genevoise se situent entre $36 \%$ et $42 \%$. Selon les chiffres de santésuisse, la facturation totale pour l'activité ambulatoire en 2000 dans le canton de Genève est de 472,8 millions. La chirurgie ambulatoire qui ressort de l'échantillon analysé représente 36 millions par année, soit $8 \%$ de l'activité ambulatoire du canton. L'étude conclut que le respect de la neutralité des coûts, en tenant compte de la chirurgie ambulatoire, implique une augmentation de la valeur du point d'environ 5\% par rapport à une étude précédente qui n'avait pas suffisamment pris en compte la chirurgie ambulatoire. Suite à une introduction du TARMED à 1 franc le point, la diminution totale de la facture ambulatoire pour les trois partenaires que sont l'opérateur, l'anesthésiste et l'hôpital, serait de 39\% par rapport aux montants facturés aujourd'hui dans le canton de Genève. Cette perte se répartirait de manière différente selon que les prestaires conservent ou non la composante technique de leurs actes.

Selon les auteurs de cette étude, les résultats présentés confirmeraient que le TARMED défavorise les prestations chirurgicales ambulatoires par rapport à d'autres activités au point d'en compromettre la poursuite.

A noter encore qu'à notre connaissance, cette étude n'a pas été publiée autre part que sur le site internet de la FMS: http://www.tarmedfms.ch/ fmstarmed/docs/RapportFinalTarmed11GEV2. pdf. Il s'agit donc de toute évidence d'un article soumis à aucun peer-review et qui est à prendre avec la réserve nécessaire dans ce genre de cas. 